\title{
Investigation of Galactosylated Low Molecular Weight Chitosan-Coated Liposomes for Cancer Specific Drug Delivery
}

\author{
Nitin K Jain ${ }^{1}$, M Chaurasia ${ }^{2}$ and SK Jain ${ }^{1^{*}}$ \\ ${ }^{1}$ Department of Pharmaceutical Sciences, Dr. Hari Singh Gour University, Sagar (M. P.) 470003, ${ }^{2}$ Amity Institute of Pharmacy, \\ Amity University, Lucknow (U. P.) 226010, India
}

*For correspondence: Email: drskjainin@yahoo.com; Tel: 0091-7582-265457; Fax: 0091-7582-264236

Revised accepted: 21 March 2014

\begin{abstract}
Purpose: To assess the hepatocyte targeting potential of galactosylated low molecular weight chitosan (Gal-LMWCs)-coated liposomes bearing doxorubicin hydrochloride (DOX).

Methods: Chitosan (CS) was depolymerized and lactobionic acid (LA) containing a galactose group was coupled with low molecular weight chitosan (LMWC) using carbodiimide chemistry. Two types of galactosylated polymers with variable degree of substitution were synthesized. Liposomes were prepared using film casting method, coated with the synthesized polymers and characterized for vesicle shape and size, polydispersity, zeta potential, drug entrapment, coating efficiency, in vitro drug release and cytotoxicity on human hepatoma cell line $\left(\mathrm{HepG}_{2}\right)$.

Results: Coating efficiency was greater for the polymer with a lower degree of substitution. The liposomes formed were spherical in shape with a size range of $110-160 \mathrm{~nm}$, drug entrapment of $92.14-96.37 \%$ and zeta potential of 20.6 - $29.4 \mathrm{mV}$. Gal-LMWC(s)-coated liposomes exhibited a maximum of $65 \%$ in vitro drug release in $24 \mathrm{~h}$ in a sustained fashion. The $50 \%$ inhibitory concentration $\left(I C_{50}\right)$ values for liposomal formulations and drug solution were 2.81 and $5.98 \mu \mathrm{g} / \mathrm{ml}$, respectively.

Conclusion: Gal-LMWC (s) coated liposomes containing DOX that demonstrate targetability to human hepatocellular carcinoma cell line (in vitro) have been successfully developed.
\end{abstract}

Keywords: Targeted delivery, Doxorubicin, Galactosylated, Chitosan, Hepatocellular, Carcinoma, Nanoparticles

Tropical Journal of Pharmaceutical Research is indexed by Science Citation Index (SciSearch), Scopus, International Pharmaceutical Abstract, Chemical Abstracts, Embase, Index Copernicus, EBSCO, African Index Medicus, JournalSeek, Journal Citation Reports/Science Edition, Directory of Open Access Journals (DOAJ), African Journal Online, Bioline International, Open-J-Gate and Pharmacy Abstracts

\section{INTRODUCTION}

Hepatocellular carcinoma (HCC) is a common primary liver cancer. Globally, HCC is the fifth most common malignancy and the third largest cause of cancer deaths [1]. In HCC, chemotherapy can be used as potential neoadjuvant and adjuvant approaches. Resistance to chemotherapy is a major obstacle and the mechanisms involved in drug resistance are complex, multifactorial and may be due to inadequate drug exposure or alterations in the cancer cell itself. Unfortunately systemic chemotherapy lacks efficacy for HCC and there is currently no standard treatment for patients with non resectable $\mathrm{HCC}$ [2].

In a metaanalysis chemoembolization, DOX was shown to improve survival of patients with advanced HCC [3] but fewer than $20 \%$ of patients respond to treatment with DOX. The anti-cancer drug may be selectively delivered by anchoring of ligand to nanocarriers that specifically interacts with receptors expressed on 
the cell surface of interest. These ligands include antibodies, glycolipids, glycoproteins, polysaccharides, proteins, and immunoregulatory molecules [4]. Parenchymal liver cells (hepatocytes) possess large numbers of highaffinity cell-surface asialoglycoprotein receptors (ASGPRs) that can bind asialoglycoproteins (ASGP). HCC showing increased expression of ASGPRs in comparison to the surrounding liver tissue has been described in humans [5]. The ASGPRs can recognize terminal $\beta$-D-galactose or $\mathrm{N}$ - acetylgalactosamine residue. Several sugar ligands (e.g., galactose, $\mathrm{N}$-acetylgalactosamine, mannose, lactose, fructose, etc) and oligosaccharides/polysaccharides (e.g., dextran) have been demonstrated to possess different extent of interaction with ASGPRs [6]. Various drug delivery systems including liposomes and nanoparticles have been reported to overcome multi drug resistance phenomena occurring at both the cellular and the non-cellular level and the results are well documented with anti-tumor drugs such as DOX [7].

Researchers have reported the synthesis of a novel galactosylated lipid with mono-galactoside moiety, (5-Cholesten-3b-yl) 4-oxo-4-[2(lactobionyl amido) ethylamido] butanoate (CHSED-LA), and the targetability of DOX in liposomes containing $10 \% \mathrm{~mol} / \mathrm{mol}$ CHS-ED-LA [8]. Majority of the colloidal carriers have a negative surface charge and therefore, they interact with the cationic biologic compounds upon in vivo administration. To impart stability to the liposomal system and to make it cationic, coating of the liposomal vesicles with a cationic polymer may be a useful strategy. Chitosan is a biocompatible, biodegradable and nontoxic polymer with cationic amino group that could be used to coat the lipid bilayer. Chitosan have been used as liposome coating material to increase the stability towards drug release and for targeting purposes. Chitosan coated liposomes have been formed via ionic interaction between the positively charged chitosan and negatively charged lipid on the surface of the liposomes [9].

The aim of the present investigation was to develop and characterize DOX-bearing, ligand anchored targeted drug delivery systems using a natural polysaccharide, chitosan and its derivatives, for effective management of HCC.

\section{EXPERIMENTAL}

\section{Materials}

Chitosan was provided by Central Marine Fisheries Research Institute, Kochi, India. Doxorubicin hydrochloride (DOX) was generously provided by $\mathrm{M} / \mathrm{s}$ Khandalwal Labs, Mumbai, India. 1-ethyl-3-(3-dimethylaminopropyl)-carbodiimide (EDC) and N, N, N', N"- tetramethylethylenediamine (TEMED) buffer solution were procured from Himedia, Mumbai, India. Lactobionic acid (LA), Phosphatidyl choline (PC), cholesterol ( $\mathrm{CH}$ ) and Phosphatidyl glycerol (PG) were obtained from Sigma, St. Louis, MO, USA. All other chemicals used were of analytical grade and used without further purification.

\section{Depolymerization of chitosan and its galactosylation}

High molecular weight chitosan (minimum 85\% deacetylated) was depolymerized using potassium persulfate followed by galactosylation by first dissolving $0.25 \mathrm{~g}$ of low molecular weight chitosan (LMWC, Mv $\approx 21 \mathrm{kDa}$ ) in $10 \mathrm{ml}$ of 10 mM N, N, N', N"- tetramethylethylenediamine (TEMED) buffer solution in a conical flask and $\mathrm{pH}$ was adjusted to 4.7 [10]. To this solution, $0.450 \mathrm{~g}$ of EDC was added and the resultant solution was stirred using a magnetic stirrer at $25^{\circ} \mathrm{C}$ for $24 \mathrm{~h}$. Different quantities of LA $\{0.179 \mathrm{~g}(0.5 \mathrm{mmol})$ and $0.268 \mathrm{~g}(0.75 \mathrm{mmol})\}$ were added to this solution and both solutions were stirred for another $72 \mathrm{~h}$ at $25^{\circ} \mathrm{C}$ on magnetic stirrers. After $72 \mathrm{~h}$ of stirring, the resulting Gal-LMWC(s) were dialyzed (12-kDa cutoff dialysis membrane) for 4 days against Milli $Q$ water and finally the purified Gal-LMWC(s) were lyophilized. The GalLMWC(s) were characterized using infrared spectroscopy (FTIR, Shimadzu 8201 PC) and by measurement of degree of substitution.

\section{Preparation of LMWC- and Gal-LMWC-coated liposomes}

The polymer coated liposomes bearing DOX, were prepared in two steps using cast film method. Phosphatidyl choline (PC), cholesterol $(\mathrm{CH})$ and phosphatidyl glycerol (PG) were dissolved in different molar ratios, in a mixture of chloroform and methanol $(2: 1 \mathrm{v} / \mathrm{v})$ to get total lipid concentration of $5 \mathrm{mg} / \mathrm{ml}$ in a round bottom flask. The mixture was evaporated to dryness in a rotary evaporator at $40^{\circ} \mathrm{C}$ and the lipid film was further dried at room temperature under vacuum overnight to completely remove the solvent. The thin lipid film was hydrated at room temperature with $10 \mathrm{ml}$ of PBS ( $\mathrm{pH} 7.4)$ for $60 \mathrm{~min}$ followed by vortexing and left for next $6 \mathrm{~h}$ for further swelling to get multilamellar vesicles (MLVs). The MLVs were sonicated to get small unilamellar vesicles (SUVs) with appropriate size. The technique reported by Kikuchi et al [11] was followed with some modification to entrap the positively charged DOX into SUVs (anionic lipid) in order to achieve more efficient drug entrapment [11]. 
In the second step, the method reported by Gonzalez-Rodriguez et al. with some modifications was used to coat the liposomes (SUVs) [12]. Briefly, LMWC and Gal-LMWC(s) $(0.025,0.05$ and $0.075 \% \mathrm{w} / \mathrm{v})$ were dissolved in a solution containing $0.2 \% \mathrm{v} / \mathrm{v}$ of glacial acetic acid and the polymer solution was added drop wise in the equal volume of suspension containing SUVs bearing drug, under controlled magnetic stirring (100 rpm). The whole system was incubated at $10 \pm 1^{\circ} \mathrm{C}$ for $30 \mathrm{~min}$. The LMWC and GalLMWC(s) coated SUVs were separated by ultra centrifugation at $20,000 \mathrm{rpm}$ at $4^{\circ} \mathrm{C}$ for $45 \mathrm{~min}$ from the liquid phase containing residual polymer. The pellet was washed with PBS $(\mathrm{pH}$ 7.4) three times, and vesicular suspension was extruded through $0.2 \mu \mathrm{m}$ polycarbonate membrane.

\section{Optimization}

Various process and formulation variables that could affect formulation characteristics and performance were studied and optimized to achieve liposomal formulation with appropriate size and maximum drug entrapment efficiency. Initially, the ratio of $\mathrm{PC}$ and $\mathrm{CH}$ was optimized to obtain appropriate vesicle size and entrapment efficiency of drug. To optimize the molar ratio of the 3rd lipid, i.e., PG, the formulations were prepared with increasing molar ratio of $P G$ and 7:3 molar ratio of $\mathrm{PC}$ : $\mathrm{CH}$. The size of the liposomes was further reduced using probe sonicator in order to make them suitable for hepatic delivery. The ratio of drug to total lipids was optimized on the basis of vesicle size, and maximum encapsulation efficiency. Coating of LMWC and Gal-LMWC(s) was accomplished according to the technique developed by Gonzalez-Rodriguez et al [12] with some modifications. The polymers were dissolved in purified water with the aid of $0.2 \% \mathrm{v} / \mathrm{v}$ glacial acetic acid. The optimized liposomal formulation LD3 was coated with the $0.025,0.05$, and 0.075 $\% \mathrm{w} / \mathrm{v}$ concentration of polymers and coating efficiency was determined.

\section{Vesicle size, polydispersity and zeta potential}

The vesicle size, polydispersity and zeta potential of the formulations were determined by photon correlation spectroscopy using Zetasizer (Nano ZS, Malvern UK). The formulations were appropriately diluted with PBS ( $\mathrm{pH}$ 7.4) and placed in quartz cuvette and size measurements were carried out at a scattering angle of $90^{\circ}$ with the help of software provided with the instrument.

\section{Evaluation of drug entrapment}

The polymer coated liposomes were subjected to ultra centrifugation at $40,000 \mathrm{rpm}$ at $4^{\circ} \mathrm{C}$ for $60 \mathrm{~min}$. The free drug in the supernatant was determined using UV-visible spectrophotometer (UV-1700, Shimadzu, Japan) at $481 \mathrm{~nm}$. Total drug content of the suspension was also determined. Entrapment efficiency was calculated as the ratio of drug content within the liposomes to total drug content of the suspension. The drug content within the liposome was calculated as the total drug content of the suspension minus free drug.

\section{Coating efficiency}

Coating efficiency of Gal-LMWC(s) was determined by the quantification of galactose residues remaining in the supernatant after the centrifugation of Gal-LMWC(s) coated and uncoated liposomes at $40,000 \mathrm{rpm}$ for $45 \mathrm{~min}$. The galactose content was determined using the phenol-sulfuric acid method. Briefly, the Gal-LMWC(s) coated liposomes were centrifuged and supernatant was separated from the settled liposomal pellet. The supernatant was diluted to make a volume of $50 \mathrm{ml}$. Phenol solution $(5 \% \mathrm{w} / \mathrm{v} ; 1 \mathrm{ml})$ was added to $2 \mathrm{ml}$ of the above solution followed by addition of $5 \mathrm{ml}$ of concentrated sulfuric acid. The flask was kept at room temperature for $30 \mathrm{~min}$ and the absorbance was measured at $490 \mathrm{~nm}$ using UV-Visible spectrophotometer. Similarly the amount of galactose residue in initial Gal-LMWC(s) was also determined. For the calculation of galactose concentration, standard curve was prepared using the galactose in water against the blank containing purified water, phenol and sulfuric acid. The amount of Gal-LMWC(s) coated on the vesicles was determined by the difference between the concentration of Gal-LMWC(s) in the separated supernatant and the total quantity of Gal-LMWC(s) added in coating process. The polymer used for the coating was calculated in terms of coating efficiency, using Eq 1.

Coating efficiency $(\%)=\{(A-B) / A\} 100 \ldots \ldots(1)$

where $\mathrm{A}=$ total galactose content in polymer and $\mathrm{B}$ $=$ galactose content in supernatant

\section{In vitro drug release study}

In vitro drug release from the uncoated liposomes, LMWC coated liposomes and GalLMWC(s) coated liposomes bearing drug was studied with dialysis membrane against PBS $(\mathrm{pH}$ 7.4). Liposomes free of any un-entrapped DOX was taken into a dialysis bag (MWCO 3500) and the bag was suspended in a receiver compartment containing $100 \mathrm{ml}$ of saline 
phosphate buffer $(\mathrm{pH} 7.4)$ and $0.1 \%$ Tween 80 to maintain the sink condition. Whole assembly was kept on a magnetic stirrer under continuous stirring $(75 \mathrm{rpm})$ at $37 \pm 1^{\circ} \mathrm{C}$ in the dark. Samples were withdrawn periodically and the amount of DOX released was monitored using UV-Visible Spectrophotometer at $481 \mathrm{~nm}$.

\section{In vitro cytotoxicity study}

Drug solution, liposomes, drug loaded GalLMWC(s) and LMWC coated liposomes were studied to assess their cell growth inhibition potential employing a tetrazolium dye (MTT) assay against HepG2 cells. After being cultured for $24 \mathrm{~h}$, the cells were immediately treated with increasing doses of selected formulations containing the DOX as well as increasing concentration of DOX solution in PBS ( $\mathrm{pH} 7.4)$ and incubated for another $48 \mathrm{~h}$. DOX loaded formulations were also added with excess amount of galactose. MTT assay was performed and cell viability was determined by measuring the absorbance at $540 \mathrm{~nm}$ using ELISA plate reader.

\section{Statistical analysis}

Student's $t$-test was used to assess significant difference between the cytotoxicity of liposomal formulation and drug solution with the aid of GraphPad InStat software (GraphPad Prism 5). Data were considered statistically significant at $p<$ 0.05 .

\section{RESULTS}

\section{Depolymerization and galactosylation of chitosan}

High molecular weight chitosan was depolymerized to LMWC(s). Approximately 50 $60 \%$ yield of Gal-LMWC(s) was obtained. The IR spectrum of lactobionic acid exhibited a broad absorption in the region $3400-2400 \mathrm{~cm}^{-1}$ showing the presence of $-\mathrm{OH}$ group and a distinctive band at $1740 \mathrm{~cm}^{-1}$ showing the carbonyl stretching $(\mathrm{C}=\mathrm{O})$ of carboxylic groups whereas in the IR spectra of Gal-LMWC(s), the disappearance of the carbonyl stretching of lactobionic acid is observed. Degree of substitution was calculated using elemental analysis data as 10.6 and $14.1 \%$ for Gal1-LMWC and for Gal2-LMWC, respectively.

\section{In vitro characterization of liposomes}

The results of the in vitro characterization of formulated liposomes are depicted in Table 1. It was observed that $\mathrm{PC}$ to $\mathrm{CH}$ ratio 7:3 gives the vesicles with appropriate parameters. The formulation prepared by optimized ratio of PC and $\mathrm{CH}$ has a vesicles size of $99.48 \pm 3.67 \mathrm{~nm}$ with $36.75 \pm 1.24 \%$ drug entrapment efficiency. Maximum drug entrapment efficiency (93.81 \pm $2.14 \%$ ) was observed with formulation with PC: $\mathrm{CH}$ : PG ratio of 7: 3: 4 showing a size of 106.94 $\pm 2.71 \mathrm{~nm}$. The vesicle size of the liposomes increased as the ratio of drug to total lipids increased. In the case of LD1 and LD2 formulations where the concentration of drug was $0.5 \mathrm{mg} / \mathrm{ml}$ and $1.0 \mathrm{mg} / \mathrm{ml}$, respectively, the vesicle size was found to be $95.45 \pm 3.17$ and $96.47 \pm 2.14 \mathrm{~nm}$, respectively while the drug entrapment efficiency for the same formulations was found to be $82.15 \pm 2.64$ and $94.28 \pm 2.23$ $\%$, respectively. The optimum concentration of drug was observed as $1.5 \mathrm{mg} / \mathrm{ml}$, which produced formulation LD3 with vesicles size of $99.82 \pm 2.86 \mathrm{~nm}$ and drug entrapment of $96.37 \pm$ $3.54 \%$ and was selected for further developmental procedures to produce polymer coated liposomes. The zeta potential of liposomal formulation (LD3) was -18.4 \pm 0.96 $\mathrm{mV}$, while for the polymer coated liposomes it was positive, which further confirms the formation of a polymer layer on the surface of liposomes.

Table 1: Liposomal and polymer-coated liposomal formulations

\begin{tabular}{|c|c|c|c|c|c|c|c|}
\hline $\begin{array}{l}\text { Formulation } \\
\text { code }\end{array}$ & Polymer & $\begin{array}{l}\text { Polymer } \\
\text { conc. } \\
(\% w / v)\end{array}$ & $\begin{array}{l}\text { Vesicle Size } \\
(\mathrm{nm})\end{array}$ & $\begin{array}{l}\text { Poly- } \\
\text { dispersity } \\
\text { Index }\end{array}$ & $\begin{array}{l}\text { Zeta } \\
\text { potential } \\
(\mathrm{mV})\end{array}$ & $\begin{array}{l}\text { Drug } \\
\text { entrapment } \\
(\%)\end{array}$ & $\begin{array}{l}\text { Coating } \\
\text { efficiency } \\
(\%)\end{array}$ \\
\hline $\mathrm{LD}_{3}$ & - & - & $99.82 \pm 2.86$ & 0.20 & $-18.4 \pm 0.96$ & $96.37 \pm 3.54$ & - \\
\hline $\mathrm{LD}_{3} \mathrm{LCL}_{1}$ & LMWC & 0.025 & $113.84 \pm 4.27$ & 0.12 & $24.1 \pm 1.51$ & $94.28 \pm 4.26$ & - \\
\hline $\mathrm{LD}_{3} \mathrm{LCL}_{2}$ & LMWC & 0.05 & $121.46 \pm 3.82$ & 0.14 & $27.5 \pm 1.43$ & $93.72 \pm 4.52$ & - \\
\hline $\mathrm{LD}_{3} \mathrm{LCL}_{3}$ & LMWC & 0.075 & $135.52 \pm 5.27$ & 0.17 & $29.4 \pm 1.95$ & $92.14 \pm 3.78$ & - \\
\hline $\mathrm{LD}_{3} \mathrm{GC}_{1} \mathrm{LD}_{1}$ & $\mathrm{Gal}_{1}$ LMWC & 0.025 & $117.82 \pm 4.24$ & 0.15 & $22.6 \pm 1.86$ & $94.56 \pm 3.82$ & $64.56 \pm 2.87$ \\
\hline $\mathrm{LD}_{3} \mathrm{GC}_{1} \mathrm{LD}_{2}$ & Gal $_{1-L M W C}$ & 0.05 & $128.47 \pm 3.86$ & 0.24 & $25.3 \pm 1.99$ & $94.12 \pm 3.47$ & $72.43 \pm 3.55$ \\
\hline $\mathrm{LD}_{3} \mathrm{GC}_{1} \mathrm{LD}_{3}$ & $\mathrm{Gal}_{1}$ LMWC & 0.075 & $145.61 \pm 4.33$ & 0.14 & $26.8 \pm 1.47$ & $92.49 \pm 4.21$ & $83.78 \pm 4.23$ \\
\hline $\mathrm{LD}_{3} \mathrm{GC}_{2} \mathrm{LD}_{1}$ & $\mathrm{Gal}_{2-}$ LMWC & 0.025 & $120.15 \pm 5.27$ & 0.11 & $20.6 \pm 1.14$ & $94.84 \pm 2.54$ & $55.81 \pm 1.92$ \\
\hline $\mathrm{LD}_{3} \mathrm{GC}_{2} \mathrm{LD}_{2}$ & $\mathrm{Gal}_{2-\mathrm{LMWC}}$ & 0.05 & $138.65 \pm 5.13$ & 0.16 & $22.9 \pm 1.92$ & $94.51 \pm 3.39$ & $66.92 \pm 3.41$ \\
\hline $\mathrm{LD}_{3} \mathrm{GC}_{2} \mathrm{LD}_{3}$ & $\mathrm{Gal}_{2}$ LMWC & 0.075 & $157.51 \pm 4.24$ & 0.13 & $23.7 \pm 1.71$ & $92.62 \pm 4.52$ & $78.59 \pm 3.97$ \\
\hline
\end{tabular}




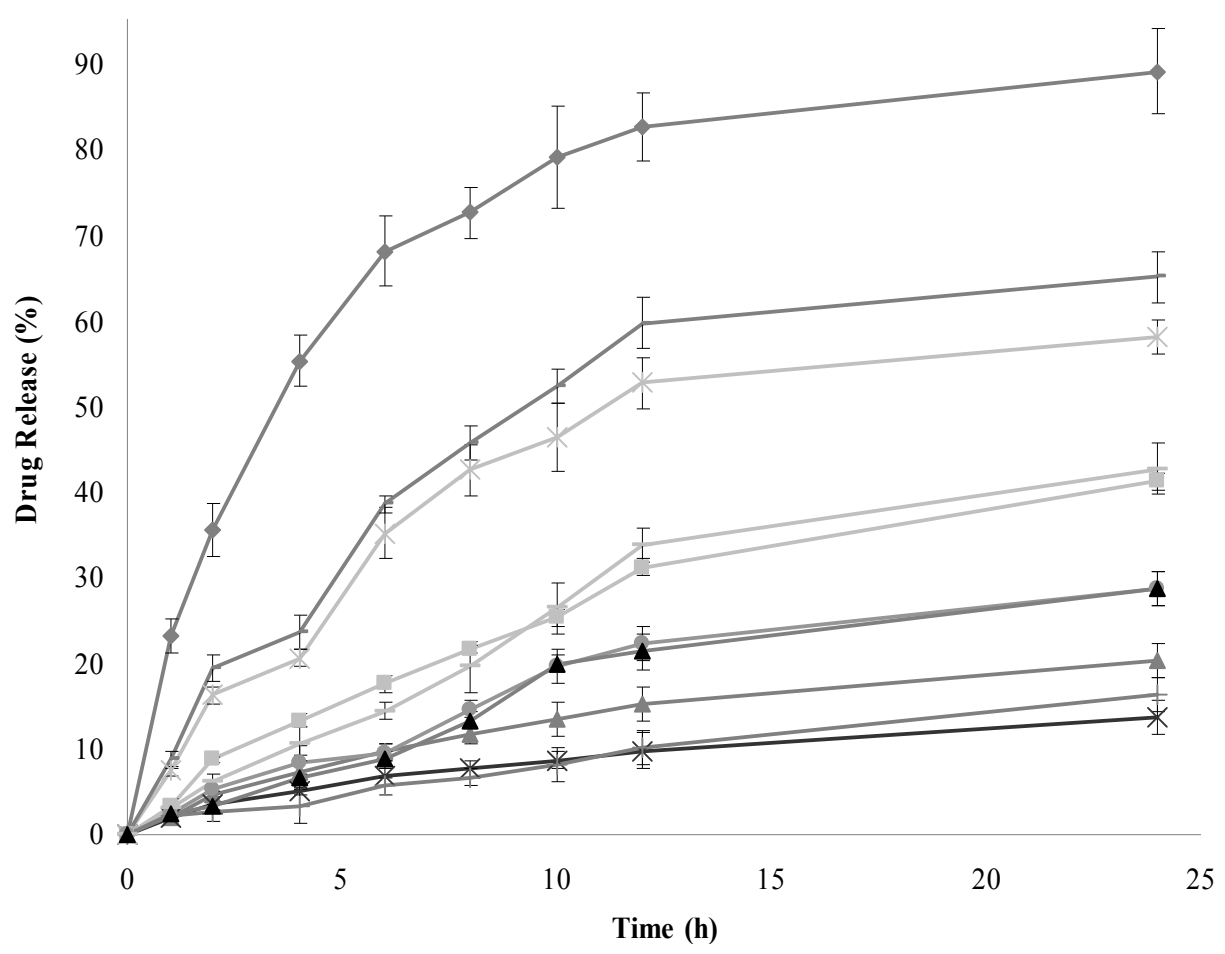

Figure 1: In vitro drug release profile of liposomal formulations. Note: $\downarrow=\mathrm{LD} 3, \boldsymbol{=}=\mathrm{LD} 3 \mathrm{LCL} 1, \boldsymbol{\Delta}=\mathrm{LD} 3 \mathrm{LCL} 2, \mathrm{X}=$ LD3LCL3, = = LD3GC1LD1, • = LD3GC1LD2, | = LD3GC1LD3, - = LD3GC2LD1, $=$ = LD3GC2LD2, $\Delta=$ LD3GC2LD3

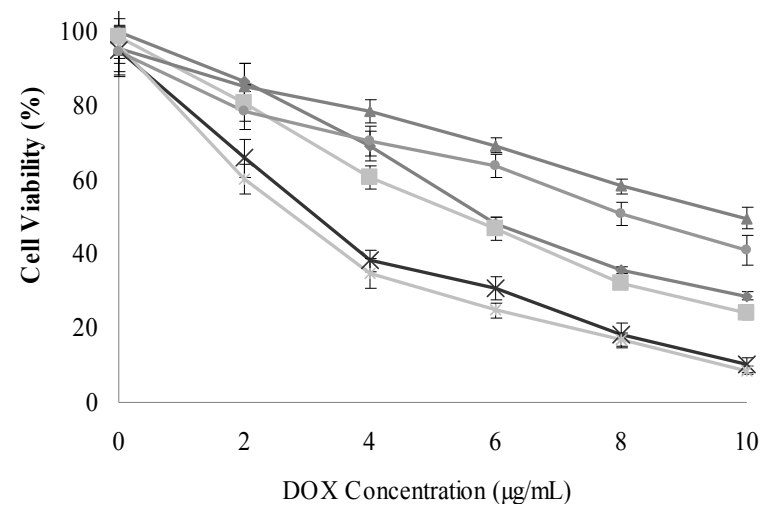

Figure 2: Cell viability (\%) of HepG2 cells after treatment with various formulations

- = Drug solution, $\mathbf{\square}=\angle D 3, \boldsymbol{\Delta}=\angle D 3 L L D 3, X=$

$L D 3 G C 1 L D 3, \neq=\angle D 3 G C 2 L D 3, \bullet=$

$L D 3 G C 2 L D 3+$ galactose

\section{In vitro drug release}

In case of uncoated liposomal formulation $\mathrm{LD}_{3}$, a rapid release of drug was observed (Figure 1 ). The cumulative drug release after $4 \mathrm{~h}$ of study was $55.36 \pm 6.14 \%$ and while cumulative drug release after $24 \mathrm{~h}$ was $89.25 \pm 3.24 \%$. In vitro drug release profile of LMWC coated liposomes and Gal-LMWC(s) coated liposomes showed entirely different release profile. After $4 \mathrm{~h}$, cumulative drug release from formulations $\mathrm{LD}_{3} \mathrm{LCL} \mathrm{L}_{1}, \mathrm{LD}_{3} \mathrm{LCL}$ and $\mathrm{LD}_{3} \mathrm{LCL}_{3}$ was found to be $13.34 \pm 1.34,7.31 \pm 0.17$ and $5.14 \pm 0.28 \%$, respectively, which shows that the coating of LMWC significantly decreases the in vitro drug release of DOX from the liposomal formulation (Figure 1). The cumulative release for formulations $L_{3} G C_{2} L D_{1}, \quad L_{3} G C_{2} L D_{2}$ and for $\mathrm{LD}_{3} \mathrm{GC}_{2} \mathrm{LD}_{3}$ was found to be 23.58 \pm 1.34 , $10.54 \pm 0.45$, and $6.53 \pm 0.43 \%$, respectively after 4 h.

\section{In vitro cytotoxicity}

The results revealed that the formulations $\mathrm{LD}_{3} \mathrm{GC}_{1} \mathrm{LD}_{3}$, and $\mathrm{LD}_{3} \mathrm{GC}_{2} \mathrm{LD}_{3}$ exhibited remarkable cytotoxic activity on the $\mathrm{HepG}_{2}$ cell line. The percent viability of $\mathrm{HepG}_{2}$ cells was found to be $10.25 \pm 0.56$ and $8.64 \pm 1.24 \%$, respectively, after incubating the cells with formulations containing $10 \mu \mathrm{g} / \mathrm{ml}$ concentration of DOX, whereas the percent viability for same concentration of drug solution was $28.72 \pm 0.54$ $\%$ (Figure 2). The higher percent viability of $\mathrm{HepG}_{2}$ cells was observed with LMWC coated liposomes $\left(L D_{3} L D L_{3}\right)$. The cell viability for the formulation $\mathrm{LD}_{3} \mathrm{GC}_{2} \mathrm{LD}_{3}$ after $24 \mathrm{~h}$ of incubation was $8.64 \pm 1.24 \%$, while in the presence of galactose the cell viability was $41.21 \pm 2.47 \%$. The $I C_{50}$ values for formulations $L D_{3} G C_{1} L D_{3}$, and $\mathrm{LD}_{3} \mathrm{GC}_{2} \mathrm{LD}_{3}$ were found to be 3.23 and 2.81 $\mu \mathrm{g} / \mathrm{ml}$ which are significantly less than the $\mathrm{IC}_{50}$ for the drug solution $(5.98 \mu \mathrm{g} / \mathrm{ml}, p<0.05)$. 


\section{DISCUSSION}

The LMWC (Mv $\approx 21 \mathrm{kDa}$ ) was successfully coupled with LA containing a galactose residue via an active ester intermediate using EDC as described by Gao et al [10]. Further IR analysis was performed to confirm the coupling of galactose residue. In the IR spectra of GalLMWC(s), the disappearance of the carbonyl stretching of lactobionic acid is observed, which could be due to the amide bond formation between carboxylic groups of lactobionic acid and the amine group of LMWC [13].

The polysaccharide anchored liposomes have paved the way for the bio-stable, site-specific and ligand directed delivery systems with desired therapeutic and immunological characteristics [14]. It has been observed that cationic liposomes are able to selectively deliver their carried load to certain tissues such as tumor endothelium, lungs and liver [15]. It was hypothesized that Gal-LMWC(s) coated liposomes could selectively be accumulated in HCC, as positive charge of amino group in polymer could be an added advantage along with the receptor mediated endocytosis of carriers by ASGPRs.

It was observed initially that increasing the molar ratio of $P G$ resulted in increase in the vesicles size and increase in drug entrapment efficiency up to $7: 3: 4$ of $\mathrm{PC}$ : $\mathrm{CH}$ : $\mathrm{PG}$, afterward further increase in $P G$ ratio resulted in nearly no change in drug entrapment. This could be due to the saturation of bilayer. The polydispersity index was found to be decreased with increasing the PG molar ratio up to 4 and further enhancement in $P G$ molar ratio caused increase in polydispersity index. This could be due to the saturation of bilayer with $P G$ at molar ratio of 4 and further raising the $P G$ molar ratio might have caused the formation of bilayers of PG with variable size, as PG itself forms the liposomes. Therefore, ratio 7:3:4 was considered optimum ratio of lipids, i.e., $\mathrm{PC}, \mathrm{CH}$ and $\mathrm{PG}$ for the preparation of liposomes.

Hashida et al reported that the majority of the fenestrate of the liver sinusoid is usually smaller than $200 \mathrm{~nm}$ in diameter [18]. Additionally, drug carriers with a diameter larger than $200 \mathrm{~nm}$ are readily scavenged non-specifically by monocytes and the reticuloendothelial system. Thus, large particles hardly reach the liver's parenchymal cells. The polydispersity index was found to be decreased with increasing sonication time up to 6 min. Further increase in sonication time caused an increase in polydispersity index that could be due to the breaking of bilayers and formation of new bilayers of variable size. The sonication time of 6 min was considered optimum as it produced formulation LS3 with $94.28 \pm 2.23 \%$ drug entrapment and $96.47 \pm 2.14 \mathrm{~nm}$ size with least polydispersity.

The small sizes of chitosan coated liposomes are produced at a low $\mathrm{pH}$, where liposomes have the lowest negative charge [16]. When liposomes were added to the solution with an excess of chitosan polymer, the chitosan adhered to the liposomal surface and polymer coated vesicles were produced. In our study we added polymer solution drop wise into liposomal suspension under constant stirring to minimize the exposure of the vesicles to acetic acid, which was used to dissolve the polymers. When polymer solution was added to the colloidal dispersion, the polymer adhered to the liposomal surface. The uniform coating of the polymer was obtained and the coated layer was not desorbed during washing. The electrostatic attraction between the positively charged amino group of polymers and opposite charge on liposomes could be the prime reason for coating, but involvement of some other mechanism in regulation of coating process could also be possible.

The increase in vesicles size after coating with LMWC confirms the formation of LMWC layer on liposomes. The vesicles size of all the formulations coated with Gal-LMWC(s) was also found to be greater than the uncoated formulation. The polydispersity index for the formulation was found to be reasonably low, which shows narrow size distribution. The increase in the vesicle size of polymer coated liposomes with increasing the concentration of polymers could be due to increase in thickness of polymer coat on liposomes. Earlier the increase was reported to be probably due to a combination of adsorption coagulation and bridging between chitosan and liposomes [19].

The zeta potential increased with increasing concentrations of polymer. Gal-LMWC(s) coated liposomes, presumably due to the presence of higher positive charge density and presence of more numbers of protonated amino groups on LMWC. It was observed that zeta potential of LMWC and Gal-LMWC(s) coated liposomes increased with increasing the concentration of polymer. Increase in polymer concentration from 0.05 to $0.075 \% \mathrm{w} / \mathrm{v}$ increased zeta potential relatively little which may be due to saturation of liposomal bilayer with the cationic polymer.

Drug entrapment data revealed that entrapment efficiency for all polymer-coated liposomes is little lower than the entrapment for the optimized 
liposomal formulation. This could be due to the leakage of drug from the liposomal formulation during the coating process. DOX is a water soluble basic drug that was added to the aqueous compartment of liposomes. The pKa value of DOX is 8.22 and therefore, a high percentage of the molecules are expected to be charged in neutral and acidic $\mathrm{pH}$. The ionized DOX fraction is localized in the membrane bilayer because of its amphiphilic nature. Under such conditions, the drug could have bonded avidly to the liposomal membrane containing negatively charged lipid, PG. The polymers (LMWC and Gal-LMWC(s)) that are also ionized significantly compete with DOX in their binding to PG, which could result in the release of some drug from the liposomal vesicle during polymer coating. This type of competition has already been reported by Guo et al for leuprolide [20]. The drug loss during coating was more prominent for the coating with LMWC, which is due to the presence of a more protonated amino group in LMWC.

The increased coating efficiency for $\mathrm{Gal}_{1} \mathrm{LMWC}$ formulations could be due the presence of a more protonated amino group in the polymer, because of low degree of substitution (10.6) than $\mathrm{Gal}_{2} \mathrm{LMWC}$ (14.1).

The uncoated liposomal formulation $\mathrm{LD}_{3}$, showed a rapid release of drug. The rapid in vitro drug release from formulation $L^{2}$ is due to greater membrane permeability of drug and mutual repulsion between the drug ions inside the liposomal vesicles. The in vitro drug release of LMWC-coated liposomes and Gal-LMWC(s)coated liposomes showed entirely different release profiles. After $4 \mathrm{~h}$, the coating of LMWC significantly decrease in vitro release of DOX from the liposomal formulation. Increase in coating thickness and the lower solubility of LMWC in the dissolution media at $\mathrm{pH} 7.4$ might be responsible for the decreased drug release.

The liposomal formulations $\mathrm{LD}_{3}, \mathrm{LD}_{3} \mathrm{LCL}_{3}$, $\mathrm{LD}_{3} \mathrm{GC}_{1} \mathrm{LD}_{3}$ and $\mathrm{LD}_{3} \mathrm{GC}_{2} \mathrm{LD}_{3}$ were selected to assess the in vitro cytotoxicity on $\mathrm{HepG}_{2}$ cell line. $\mathrm{HepG}_{2}$ cell line is human hepatocellular carcinoma cell line expressing the ASGP receptors on their surfaces. The greater viability of $\mathrm{HepG}_{2}$ cells observed for LMWC-coated liposomes $\left(\mathrm{LD}_{3} \mathrm{LDL}_{3}\right)$ could be due to sustained release of drug from the formulation. The liposomal formulation $\mathrm{LD}_{3}$ exhibited comparable cytotoxicity with the plain drug solution, which is due to the rapid release of drug from the formulation. Given the limited in vitro drug release exhibited by the formulations, $\mathrm{LD}_{3} G \mathrm{C}_{1} \mathrm{LD}_{3}$, and $\mathrm{LD}_{3} \mathrm{GC}_{2} \mathrm{LD}_{3}$, after $24 \mathrm{~h}$, it is hypothesized that the cytotoxic action exhibited by these ligand carriers could be due to endocytosis, rather than the release of free drug in the cell culture medium.

Further, to examine whether GalLMWC(s) coated liposomes are taken up by asialoglycoprotein receptors, the competitive inhibition experiment was performed. The cell viability of $\mathrm{HepG}_{2}$ cells markedly increased when formulation $\mathrm{LD}_{3} \mathrm{GC}_{2} \mathrm{LD}_{3}$ was incubated with an excess of galactose, suggesting the formulation was effectively taken up by asialoglycoprotein receptors in $\mathrm{HepG}_{2}$ cells. The results obtained indicate that the chemosensitivity of $\mathrm{HepG}_{2}$ cells for DOX substantially increased with GalLMWC(s) coated liposomes of appropriate size.

\section{CONCLUSION}

Galactosylated low molecular weight chitosan coated liposomes containing DOX can be suitably prepared with good physicochemical properties. The vesicles are spherical, of suitable size and zeta potential and demonstrated targetability on human hepatocellular carcinoma cell line expressing ASGP receptors on their surfaces.

\section{ACKNOWLEDGEMENT}

The authors would like to thank the Head, Department of Pharmaceutical Sciences, Dr. Hari Singh Gour University, Sagar for providing facilities to carry out the work.

\section{REFERENCES}

1. Bosch FX, Ribes J, Diaz M, R. C. Primary liver cancer: worldwide incidence and trends. Gastroenterology. 2004; 127: S5-S16.

2. Llovet JM, Bruix J. Systematic review of randomized trials for unresectable hepatocellular carcinoma: Chemoembolization improves survival. Hepatology. 2003; 37: 429-442.

3. Baird RD, Kaye SB. Drug resistance reversal-are we getting closer ? Eur J Cancer. 2003; 39: 2450-61.

4. Karra N, Benita $S$. The ligand nanoparticle conjugation approach for targeted cancer therapy. Curr Drug Metab. 2012; 13: 22-41.

5. Suzuki A, Togashi $H$, Haga $H$, Saito $K$, Saito $T$, Takahashi $K$, et al. Characteristics of three cases of hepatocellular carcinoma showing enhanced technetium-99m-diethylenetriaminepentaacetic acid-galactosyl human serum albumin accumulation by single photon emission computed tomography analysis. . Hepatol Res. 2007; 37: 628636.

6. Lin WJ, Chen MH. Synthesis of multifunctional chitosan with galactose as a targeting ligand for glycoprotein receptor. Carbohydr Polym. 2007; 67: 474-480.

7. Muggia FM, Hainsworth JD, Jeffers $S$, Miller P, Groshen $S$, Tan $M$, et al. Phase II study of liposomal doxorubicin in refractory ovarian cancer: antitumor activity and toxicity modification by liposomal encapsulation. J Clin Oncol. 1997; 15: 987-993. 
8. Wang SN, Deng YH, Xu H, Wu HB, Qiu YK, Chen DW. Synthesis of a novel galactosylated lipid and its application to the hepatocyte-selective targeting of liposomal doxorubicin. Eur J Pharm Biopharm. 2006; 62: 32-38.

9. Kozlova NO, Bruskovskaya IB, Okuneva IB, MelikNubarov NS, Yaroslavov AA, Kabanov VA, et al. Interaction of a cationic polymer with negatively charged proteoliposomes. Biochim Biophys Acta. 2001; 1514: 139-151.

10. Gao S, Chen J, Xu X, Ding Z, Yang YH, Hua Z, et al. Galactosylated low molecular weight chitosan as DNA carrier for hepatocyte-targeting. Int J Pharm. 2003; 255: 57-68.

11. Kikuchi H, Yachi K, Morita H, Hirota S. Method of producing liposomal products from freeze or spraydried preparations of liposomes. Daiich Pharmaceutical Co, Ltd, US Patent no. 5,376,380, December 27, 1994

12. Gonzalez-Rodriguez ML, Barros LB, Palma J, Gonzalez-Rodriguez PL, Rabasco AM. Application of statistical experimental design to study the formulation variables influencing the coating process of lidocaine liposomes. Int J Pharm. 2007; 337: 336-345.

13. Jain NK, Jain SK. Development and in vitro characterization of galactosylated low molecular weight chitosan nanoparticles bearing doxorubicin AAPS PharmSciTech. 2010; 11: 686-697.
14. Sihorkar V Vyas SP. Potential of polysaccharide anchored liposomes in drug delivery, targeting and immunization. J Pharm Pharm Sci. 2001; 4: 138158.

15. Dass CR, Choong PFM. Journal of Controlled Release. Selective gene delivery for cancer therapy using cationic liposomes: In vivo proof of applicability. 2006; 113: 155-163.

16. Henriksen I, Smistad G, Karlsen J. Interactions between Liposomes and Chitosan. Int J Pharm. 1994; 101: 227-236.

17. Henriksen I, Vagen SR, Sande SA, Smistad G, Karlsen $J$. Interactions between liposomes and chitosan 2. Effect of selected parameters on aggregation and leakage. Int J Pharm. 1997; 146: 193-203.

18. Hashida M, Takemura S, Nishikawa M, Takakura Y. Targeted delivery of plasmid DNA complexed with galactosylated poly(L-lysine). J Control Release. 1998; 53: 301-310.

19. Filipovic-Grcic J, Skalko-Basnet $N$, Jalsenjak $I$. Mucoadhesive chitosan-coated liposomes: characteristics and stability. J Microencapsul. 2001; 18: 3-12.

20. Guo J, Ping Q, Jiang G, Huang L, Tong Y. Chitosancoated liposomes: characterization and interaction with leuprolide. Int J Pharm. 2003; 260: 167-173. 